BMJ Open

Diabetes

Research

\& Care

\title{
Prevalence of bone fracture and its association with severe hypoglycemia in Japanese patients with type 1 diabetes
}

\author{
Yuji Komorita (D) , ,2 Masae Minami, ${ }^{2,3}$ Yasutaka Maeda, ${ }^{2,3}$ Rie Yoshioka, ${ }^{2,3}$ \\ Toshiaki Ohkuma, ${ }^{1}$ Takanari Kitazono ${ }^{1}$
}

To cite: Komorita Y, Minami M, Maeda Y, et al. Prevalence of bone fracture and its association with severe hypoglycemia in Japanese patients with type 1 diabetes. BMJ Open Diab Res Care 2021;9:e002099. doi:10.1136/ bmjdrc-2020-002099

- Supplemental material is published online only. To view, please visit the journal online (http://dx.doi.org/10.1136/ bmjdrc-2020-002099).

Received 25 December 2020 Revised 9 March 2021 Accepted 5 April 2021

Check for updates

(C) Author(s) (or their employer(s)) 2021. Re-use permitted under CC BY-NC. No commercial re-use. See rights and permissions. Published by BMJ.

${ }^{1}$ Department of Medicine and Clinical Science, Graduate School of Medical Sciences, Kyushu University, Fukuoka, Japan

${ }^{2}$ Minami Diabetes Clinical Research Center, Fukuoka, Japan

${ }^{3}$ Clinic Masae Minami,

Fukuoka, Japan

Correspondence to Dr Yuji Komorita; yjkmrt@intmed2.med.kyushuu.ac.jp

\section{ABSTRACT}

Introduction Type 1 diabetes (T1D) is associated with higher fracture risk. However, few studies have investigated the relationship between severe hypoglycemia and fracture risk in patients with T1D, and the results are controversial. Besides, none has investigated the risk factors for fracture in Asian patients with T1D. The aim of the present study was to investigate the prevalence of bone fracture and its relationship between severe hypoglycemia and other risk factors in Japanese patients with T1D.

Research design and methods The single-center crosssectional study enrolled 388 Japanese patients with T1D (mean age, 45.2 years; women, 60.4\%; mean duration of diabetes, 16.6 years) between 0ctober 2019 and April 2020. The occurrence and circumstances of any fracture after the diagnosis of T1D were identified using a selfadministered questionnaire. The main outcomes were any anatomic site of fracture and fall-related fracture. Severe hypoglycemia was defined as an episode of hypoglycemia that required the assistance of others to achieve recovery. Results A total of 92 fractures occurred in 64 patients, and 59 fractures $(64 \%)$ were fall-related. Only one participant experienced fracture within the 10 years following their diagnosis of diabetes. In logistic regression analysis, the multivariate-adjusted $0 \mathrm{Rs}(95 \% \mathrm{Cls})$ of a history of severe hypoglycemia were 2.11 (1.11 to 4.09 ) for any fracture and 1.91 (0.93 to 4.02) for fall-related fracture. Fourteen of 18 participants with multiple episodes of any type of fracture had a history of severe hypoglycemia ( $p<0.001$ vs no fracture).

Conclusions We have shown that a history of severe hypoglycemia is significantly associated with a higher risk of bone fracture in Japanese patients with T1D.

\section{INTRODUCTION}

Although the number of patients with type 1 (T1D) or type 2 (T2D) diabetes is increasing worldwide,${ }^{12}$ recent advances in the treatment of diabetes and its vascular complications have prolonged life expectancy. ${ }^{34}$ Therefore, a better understanding is required of classical microvascular and macrovascular disease, and of aging-related complications, such as malignancy, cognitive dysfunction, or bone fracture. ${ }^{56}$ Both T1D and T2D have been reported to be associated with higher risks of

\section{Significance of this study}

What is already known about this subject?

- To date, no study has investigated the prevalence and risk factors for bone fracture in Asian patients with type 1 diabetes (T1D).

- Longer duration of diabetes, poor glycemic control, and diabetic complications have been shown to increase fracture risk in T1D.

- Hypoglycemia is also considered to be one of the major risk factors for fracture in T1D, although the epidemiological findings have been contradictory in previous studies.

What are the new findings?

- A history of severe hypoglycemia was significantly associated with a higher risk of bone fracture (multivariate-adjusted OR 2.11 (95\% Cl 1.11 to 4.09)), even after adjustment for the duration of diabetes and diabetic microvascular complications.

- We have measured the prevalence and identified the characteristics of bone fracture in Asian patients with T1D.

\section{How might these results change the focus of} research or clinical practice?

- Careful treatment for the prevention of diabetic complications and of severe hypoglycemia should be needed to reduce fracture risk in patients with T1D.

bone fracture. ${ }^{78}$ In particular, the risk of fracture has been shown to be twofold to sixfold higher in individuals with T1D, compared with individuals without. ${ }^{78}$ Because bone fracture, and especially fragility fracture, is associated with impaired quality of life and higher mortality, ${ }^{910}$ it is important that the mechanisms involved and risk factors for fracture in T1D are further studied.

The pathogenesis of T1D-associated fractures appears to be multifactorial, and to involve impairment in osteoblastic bone formation caused by insulin deficiency, long exposure to glucose toxicity, other metabolic alterations, and/or a higher risk of falls because of diabetic complications. ${ }^{611}$ To 
date, epidemiological studies have shown that a longer duration of diabetes, poor glycemic control, and diabetic complications increase fracture risk in T1D. ${ }^{12-15}$ Hypoglycemia is also considered to be one of the major risk factors for fracture in T1D, although the epidemiological findings have been contradictory: some previous studies have found a significant association between hypoglycemia and fracture risk, ${ }^{16}{ }^{17}$ whereas others have failed to show this. ${ }^{13} 1418$

Inconsistency in the previous findings regarding hypoglycemia and fracture risk may be partly due to inconsistent definitions of hypoglycemia and fracture outcomes. Because the prevalence of T1D is much lower than that of T2D, ${ }^{19}$ the previous studies were of patients with T1D from a number of institutions, at which medical standards may have differed. In particular, the incidence of T1D in Asia is lower than in Western countries, ${ }^{19}{ }^{20}$ but Asia has a large population, accounting for $60 \%$ of the global population. ${ }^{21}$ To date, there has been no investigation of the prevalence of or risk factors for bone fracture in Asian patients with T1D. Therefore, we aimed to investigate the relationship between severe hypoglycemia, other risk factors, and fracture risk in Japanese patients with T1D at a single institution.

\section{RESEARCH DESIGN AND METHODS \\ Study participants}

This single-center, cross-sectional study was conducted at Clinic Masae Minami (Fukuoka, Japan), which is certified by the Japan Diabetes Society. Patients aged $\geq 20$ years who had T1D and attended the clinic between October 2019 and April 2020 were invited to participate in the study. A diagnosis of T1D was made on the basis of the Japan Diabetes Society criteria. ${ }^{22}$ Briefly, the criteria were permanent insulin treatment and/or positivity for markers of autoimmune destruction, such as glutamic acid decarboxylase antibody, and these are almost identical to the criteria of the American Diabetes Association. ${ }^{23}$ A total of 389 patients with T1D were initially enrolled, but 1 patient who did not answer the question regarding fracture was excluded, such that 388 participants remained.

\section{Clinical evaluation and laboratory measurements}

The participants completed a self-administered questionnaire regarding their smoking habits, alcohol drinking habits, exercise habits, family history of hip fracture, maximum body mass, and history of falls or severe hypoglycemia. Participants were classified as either current smokers or not, and alcohol drinkers were defined as those who drank $\geq 5$ days a week. Participants who engaged in daily exercise, including gymnastics were placed in a 'regular exercise' group. A history of falls was defined as at least one fall within the preceding year. Body mass index (BMI) was calculated using body mass and height. Weight loss was calculated by subtracting the body mass of the participant at the time of completing the questionnaire from their maximum body mass, and was expressed as a percentage of the maximum body mass. ${ }^{24}$ Blood pressure was measured in the sitting position using a standardized automated device. The participants' clinical records were reviewed for the age of onset of their diabetes, history of hypertension, dyslipidemia, and other comorbidities.

Severe hypoglycemia was defined as an episode of hypoglycemia that required the assistance of others to achieve recovery, ${ }^{25}$ and participants who had experienced at least one episode after a diagnosis of T1D were placed in a 'severe hypoglycemia' group.

Hemoglobin $\mathrm{A}_{1 \mathrm{c}}\left(\mathrm{HbA}_{1 \mathrm{c}}\right)$ was measured at the clinic by high-performance liquid chromatography, and is expressed as National Glycohemoglobin Standardization Program values. ${ }^{26}$ Non-fasting blood samples were collected byvenipuncture and were used to measure serum creatinine by an enzymatic method and serum albumin by the bromocresol purple method. $\mathrm{HbA}_{1 \mathrm{c}}$ is expressed as the mean value over the preceding 12 months. The serum creatinine and serum albumin concentrations shown are the most recent values collected during the preceding 3 years. Estimated glomerular filtration rate (eGFR) was calculated using the serum creatinine concentration and the equation proposed by the Japanese Society of Nephrology. ${ }^{27}$

Diabetic neuropathy was diagnosed using the simplified criteria proposed by the Diabetic Neuropathy Study Group in Japan, ${ }^{28}$ which specify that patients should meet any two of the following three criteria: (1) presence of symptoms considered to be due to diabetic polyneuropathy, (2) bilateral loss of or decrease in ankle reflex, and (3) decrease in bilateral vibration sensing over the medial malleoli. Diabetic retinopathy was diagnosed by an ophthalmologist, and was recorded as positive at any stage of diabetic retinopathy. Diabetic kidney disease (DKD) was defined as a urinary albumin/creatinine ratio $\geq 30 \mathrm{mg} / \mathrm{g}$ Cr or an eGFR $<60 \mathrm{~mL} / \mathrm{min} / 1.73 \mathrm{~m}^{2} \cdot{ }^{29}$ Cardiovascular disease was defined as a history of stroke or coronary artery disease, with 'stroke' including both ischemic stroke and intracranial hemorrhage, and 'coronary artery disease' including myocardial infarction, angina pectoris, and coronary intervention.

\section{Assessment of fractures}

The occurrence of any fracture after the diagnosis of T1D was identified using a self-administered questionnaire, which included the age of the participant at the time of fracture and the anatomic site of the fracture. The participants selected the sites of fractures from the following list: head/face/jaw, scapula, clavicle, arm, wrist, hand/ finger, rib/sternum, pelvis, coccyx, hip, femur without hip, knee, lower leg, ankle, foot/toe, spine, other, and unknown. Fractures that occurred during a road-traffic accident were excluded. We also assessed the circumstances of the fracture using the same questionnaire; whether the fracture was associated with a fall, hypoglycemia, or alcohol drinking. 


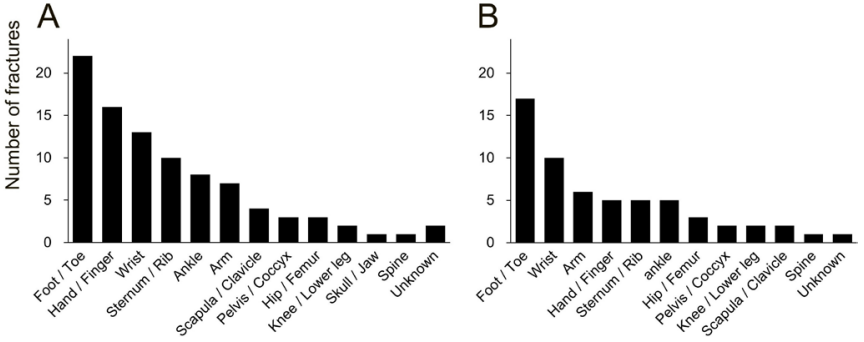

Figure 1 Frequencies of any fracture $(A)$ and fall-related fractures $(B)$ by anatomic sites after diagnosed with diabetes in Japanese patients with type 1 diabetes.

\section{Statistical analysis}

Differences in mean values or proportions between fracture groups were assessed using Student's t-test or the $\chi^{2}$ test, as appropriate. We evaluated the relationships between severe hypoglycemia, neuropathy, diabetic retinopathy, $\mathrm{DKD}$, and the history of fracture using logistic regression analysis and estimated ORs with $95 \%$ CIs. Multivariate adjustment was performed according to age, sex, BMI, duration of diabetes, smoking status, current drinking habits, exercise habits, family history of hip fracture, weight loss, and $\mathrm{HbA}_{1 \mathrm{c}}$. For the evaluation of the relationship between severe hypoglycemia and fracture, we further adjusted for the presence of neuropathy, diabetic retinopathy, and DKD. We performed the statistical analyses with Statistical Analysis Software V.9.4 (SAS Institute, Cary, North Carolina, USA). Values of $\mathrm{p}<0.05$ were considered to represent statistical significance.

\section{RESULTS}

\section{Fractures characteristics}

A total of 92 fractures occurred after a diagnosis of diabetes in 64 patients with T1D. Of these fractures, 59 $(64 \%)$ were fall-related, $6(7 \%)$ were alcohol-related and $4(4 \%)$ were hypoglycemia-related. Forty-six patients experienced a single episode of fracture and 18 patients experienced at least two episodes. Figure 1 shows the frequencies with which fracture occurred at each anatomic site (figure 1A) and the frequency with which fall-related fracture occurred at each site (figure 1B). For any fracture, foot/toe $(25 \%)$ fractures were the most frequent, followed by hand/finger $(18 \%)$, wrist $(15 \%)$, sternum/rib (11\%), ankle (9\%), and arm (8\%) fractures. There were only four fragility fractures, such as those of the hip/femur or spine. The distribution of fracture site for fall-related fractures was almost identical to that for any fracture. Online supplemental figure 1 displays the percentages for any fracture (online supplemental figure 1A) and fall-related fractures (online supplemental figure 1B) according to the duration of diabetes. Few fractures occurred in participants who had diabetes for $<10$ years, but the number of any or fall-related fractures increased with the duration of diabetes.
Clinical characteristics of participants with fracture history

Table 1 summarizes the clinical characteristics of the participants according to their history of any fracture or a fall-related fracture. Both participants who had experienced any fracture and those who had experienced fall-related fractures were more likely to have had an early onset of diabetes and to have a longer duration of diabetes. The prevalence of severe hypoglycemia and diabetic retinopathy, among patients who had experienced a fracture or a fall-related fracture, was approximately twice as high as that among patients who had not. Participants who had experienced any fracture were more likely to be male and a smoker, and to have a higher BMI, than those who had not. In contrast, participants who had experienced fall-related fractures were more likely to have fallen in the preceding 12 months, and to have a history of DKD or cardiovascular disease than those who had not.

\section{Severe hypoglycemia and fracture}

Table 2 shows the ORs for any fracture or fall-related fracture, according to the history of severe hypoglycemia. Of the participants who had experienced severe hypoglycemia, $25.9 \%$ had experienced any fracture, in contrast to only $9.7 \%$ of the participants who had not. The ageadjusted and sex-adjusted OR (95\% CI) of severe hypoglycemia for any fracture was 3.12 (1.78 to 5.62), and the multivariate-adjusted OR $(95 \% \mathrm{CI})$ was 2.10 (1.11 to 4.06). Further adjustment for the presence of neuropathy, diabetic retinopathy, and DKD did not significantly affect this finding. Of the patients who had experienced severe hypoglycemia, fall-related fractures had occurred in $19.1 \%$, in contrast to only $7.5 \%$ of the participants who had not. The age-adjusted and sex-adjusted OR (95\% CI) of severe hypoglycemia for fall-related fractures was 2.98 (1.59 to 5.79), and the multivariate-adjusted OR (95\% CI) was 1.93 (0.94 to 4.04). Online supplemental figure 2 displays the relationships between total number of fractures or the number of fall-related fractures and the history of severe hypoglycemia. Of the participants who had experienced a single episode of any fracture, $61 \%$ had a history of severe hypoglycemia, and $78 \%$ of the participants who had experienced multiple episodes of any fracture also had this history, whereas only $37 \%$ of the participants who had not experienced fracture had this history ( $\mathrm{p}$ for trend $<0.001$ (unadjusted); $\mathrm{p}$ for trend=0.02 (multivariate-adjusted)). In addition, the combination of a history of a fall and severe hypoglycemia was also significantly associated with higher risks of both any and fall-related fracture (online supplemental table 1).

\section{Diabetic complications and fracture}

The relationships between diabetic microvascular complications and fracture risk are shown in online supplemental tables 2-4. Of the participants with neuropathy, 23.0\% had a history of any fracture, whereas $14.6 \%$ without neuropathy had such a history (online supplemental 
Table 1 Clinical characteristics of patients with type 1 diabetes according to the history of any fracture or fall-related fracture

\begin{tabular}{|c|c|c|c|c|c|c|}
\hline & Any fractu & & & Fall-relate & racture & \\
\hline & $(-)$ & $(+)$ & $P$ value & $(-)$ & $(+)$ & $P$ value \\
\hline $\mathrm{N}$ & 324 & 64 & & 340 & 48 & \\
\hline Age (years) & $45.3 \pm 14.7$ & $44.9 \pm 16.9$ & 0.82 & $45.2 \pm 14.8$ & $46.2 \pm 17.0$ & 0.63 \\
\hline Female sex (\%) & 63.0 & 46.9 & 0.02 & 61.2 & 54.2 & 0.35 \\
\hline Onset of diabetes (years) & $30.2 \pm 15.7$ & $21.0 \pm 14.4$ & $<0.001$ & $29.8 \pm 15.8$ & $20.8 \pm 14.3$ & $<0.001$ \\
\hline Duration of diabetes (years) & $15.2 \pm 10.5$ & $24.0 \pm 9.4$ & $<0.001$ & $15.4 \pm 10.4$ & $25.6 \pm 9.5$ & $<0.001$ \\
\hline $\mathrm{BMI}\left(\mathrm{kg} / \mathrm{m}^{2}\right)$ & $22.5 \pm 3.3$ & $23.4 \pm 3.7$ & 0.04 & $22.6 \pm 3.3$ & $23.0 \pm 3.8$ & 0.36 \\
\hline Weight loss (\%) & $10.6 \pm 14.3$ & $8.9 \pm 11.8$ & 0.39 & $10.6 \pm 14.4$ & $8.3 \pm 9.0$ & 0.13 \\
\hline Current smoker (\%) & 13.6 & 23.4 & 0.04 & 14.4 & 20.8 & 0.25 \\
\hline Current drinker (\%) & 13.6 & 17.2 & 0.45 & 12.9 & 22.9 & 0.06 \\
\hline Exercise habit (\%) & 60.6 & 59.4 & 0.86 & 61.0 & 56.3 & 0.53 \\
\hline Fall in the past 12 months (\%) & 21.3 & 31.3 & 0.09 & 20.9 & 37.5 & 0.01 \\
\hline Family history of hip fracture (\%) & 3.1 & 4.7 & 0.52 & 3.0 & 6.3 & 0.24 \\
\hline $\mathrm{HbA}_{1 \mathrm{c}}(\%)$ & $7.7 \pm 0.9$ & $7.6 \pm 0.9$ & 0.42 & $7.7 \pm 1.0$ & $7.7 \pm 0.8$ & 0.96 \\
\hline $\mathrm{HbA}_{1 \mathrm{c}}(\mathrm{mmol} / \mathrm{mol})$ & $61.1 \pm 10.2$ & $60.0 \pm 10.2$ & 0.42 & $60.9 \pm 10.4$ & $61.0 \pm 9.0$ & 0.96 \\
\hline eGFR $\left(\mathrm{mL} / \mathrm{min} / 1.73 \mathrm{~m}^{2}\right)$ & $88.7 \pm 19.3$ & $87.4 \pm 19.4$ & 0.63 & $88.7 \pm 19.1$ & $87.4 \pm 20.4$ & 0.68 \\
\hline Serum albumin (g/dL) & $4.0 \pm 0.3$ & $4.0 \pm 0.4$ & 0.90 & $4.0 \pm 0.3$ & $4.0 \pm 0.3$ & 0.94 \\
\hline Systolic blood pressure $(\mathrm{mm} \mathrm{Hg})$ & $113 \pm 12$ & $115 \pm 10$ & 0.13 & $113 \pm 12$ & $115 \pm 10$ & 0.14 \\
\hline Diastolic blood pressure (mm Hg) & $66 \pm 8$ & $66 \pm 7$ & 0.95 & $66 \pm 8$ & $66 \pm 7$ & 0.80 \\
\hline Hypertension (\%) & 10.5 & 14.1 & 0.41 & 10.6 & 14.6 & 0.41 \\
\hline Dyslipidemia (\%) & 17.6 & 15.6 & 0.70 & 17.7 & 14.6 & 0.60 \\
\hline History of severe hypoglycemia (\%) & 37.0 & 65.6 & $<0.001$ & 38.5 & 64.6 & $<0.001$ \\
\hline Diabetic complication & & & & & & \\
\hline Neuropathy (\%) & 20.7 & 31.3 & 0.06 & 20.9 & 33.3 & 0.053 \\
\hline Diabetic retinopathy (\%) & 29.9 & 51.6 & $<0.001$ & 30.3 & 56.3 & $<0.001$ \\
\hline DKD (\%) & 17.0 & 25.0 & 0.13 & 16.8 & 29.2 & 0.04 \\
\hline Cardiovascular disease (\%) & 1.5 & 4.7 & 0.11 & 1.5 & 6.3 & 0.03 \\
\hline
\end{tabular}

Values are expressed as means \pm SD or percentages.

$\mathrm{BMI}$, body mass index; DKD, diabetic kidney disease; eGFR, estimated glomerular filtration rates; $\mathrm{HbA}_{1 \mathrm{c}}$, hemoglobin $\mathrm{A}_{1 \mathrm{c}}$.

table 2). The age-adjusted and sex-adjusted OR (95\% CI) of neuropathy for any fracture was 2.14 (1.02 to 4.51), but this finding became less significant after multivariate adjustment. Similar tendencies were observed for participants with diabetic retinopathy and DKD (online supplemental tables 3,4), and multivariate adjustment also reduced the significance of these findings. Table 3 shows the relationship between the number of diabetic microvascular complications and fracture. Compared with patients without any complications, the age-adjusted and sex-adjusted ORs (95\% CIs) for any fracture were 2.43 (1.20 to 4.92 ) in those with one complication and 4.74 (2.19 to 10.48) in those with two or more complications, although the significance diminished after multivariate adjustment, including for the duration of diabetes and severe hypoglycemia.

\section{Other factors and fracture}

Finally, we evaluated other risk factors for any fracture and fall-related fracture (table 4). A longer duration of diabetes was significantly associated with a higher risk of both any fracture and fall-related fracture. Smoking was also significantly associated with a higher risk of any fracture, whereas a family history of hip fracture was associated with a higher risk of fall-related fracture.

\section{DISCUSSION}

In this single-center, cross-sectional study, we demonstrated that severe hypoglycemia was associated with a higher risk of bone fracture in Japanese patients with T1D. The relationship between severe hypoglycemia and any fracture was found to be significant after multivariate adjustment for covariates, including the duration of diabetes, glycemic control, the presence of a diabetic complication, and smoking status. To our knowledge, this is the first study to investigate the prevalence of, and risk factors for, fracture in Asian patients with T1D. 
Table 2 Adjusted ORs and 95\% Cls for any fracture or fall-related fracture according to severe hypoglycemia in patients with type 1 diabetes

\begin{tabular}{|c|c|c|c|c|}
\hline & \multicolumn{2}{|l|}{ Any fracture } & \multicolumn{2}{|c|}{ Fall-related fracture } \\
\hline & $\begin{array}{l}\text { Without severe } \\
\text { hypoglycemia }\end{array}$ & $\begin{array}{l}\text { With severe } \\
\text { hypoglycemia }\end{array}$ & $\begin{array}{l}\text { Without severe } \\
\text { hypoglycemia }\end{array}$ & $\begin{array}{l}\text { With severe } \\
\text { hypoglycemia }\end{array}$ \\
\hline Number of patients with fracture & 22 & 42 & 17 & 31 \\
\hline Number of total patients & 226 & 162 & 226 & 162 \\
\hline Percentage of patients with fracture (\%) & 9.7 & 25.9 & 7.5 & 19.1 \\
\hline \multicolumn{5}{|l|}{ ORs $(95 \% \mathrm{Cl})$} \\
\hline Unadjusted & 1.0 (ref.) & 3.25 (1.87 to 5.78 ) & 1.0 (ref.) & 2.90 (1.57 to 5.57$)$ \\
\hline Age-adjusted and sex-adjusted & 1.0 (ref.) & 3.12 (1.78 to 5.62$)$ & 1.0 (ref.) & 2.98 (1.59 to 5.79$)$ \\
\hline Multivariate adjusted & 1.0 (ref.) & 2.10 (1.11 to 4.06$)$ & 1.0 (ref.) & $1.93(0.94$ to 4.04$)$ \\
\hline $\begin{array}{l}\text { Multivariate adjusted+neuropathy, diabetic } \\
\text { retinopathy, DKD }\end{array}$ & 1.0 (ref.) & 2.11 (1.11 to 4.09 ) & 1.0 (ref.) & 1.91 (0.93 to 4.02$)$ \\
\hline
\end{tabular}

Multivariate adjustments include age, sex, BMI, diabetes duration, current smoking habit, current drinking habit, exercise habit, family history of hip fracture, weight loss, and hemoglobin $A_{11}$.

$\mathrm{BMI}$, body mass index; DKD, diabetic kidney disease.

\section{Anatomic sites of fractures}

We have shown that the most frequent sites for fracture in patients with T1D are the foot/toe, wrist, and hand/ finger. This finding is almost identical to that of a previous study of 756 patients with T1D in the USA, in which the top three fracture sites were metatarsal/toe (24\%), metacarpal/finger (21\%), and elbow/radius/ulna/humerus $(15 \%) \cdot{ }^{30}$ Furthermore, few participants had experienced fragility fractures, such as those of the hip/femur or spine, in the present study, which was also consistent with the findings of the previous study, which recorded $5 \%$ of fractures in the hip/pelvis/femur and 3\% in the vertebrae, although the classification of fracture site in this study differed slightly from that in the present study. ${ }^{30}$ These results may be explained by the fact that the samples in the present and previous study included relatively younger participants. Only $65 \%$ of all the fractures recorded were fall-related fractures in the present study, which may imply that the remaining fractures occurred during sports or were caused by a high-energy accident. Furthermore, we may have underestimated the number of spinal fractures because only one-third of all spinal fractures are associated with clinical symptoms. ${ }^{31}$

\section{Frequency of fractures}

With respect to the number of fractures that occurred after a diagnosis of T1D, $12 \%$ of the participants had experienced a single fracture and $5 \%$ had experienced multiple fractures in the present study. This is consistent with the findings of a previous study of 600 patients with T1D in Italy (mean age, 41.9 years, mean duration of diabetes, 19.9 years) in which $14 \%$ had experienced a single fracture and $5 \%$ had experienced multiple fractures. ${ }^{13}$ Given that mean age and duration of diabetes were similar between the samples in the previous and present studies, the incidence of T1D related-fracture in Asian populations may be similar to those in Western populations, despite the fact that the incidence of T1D in Asia is lower. ${ }^{19} 20$

\section{Severe hypoglycemia and fractures in T1DM}

Only a few epidemiological studies have investigated the relationship between hypoglycemia and fracture risk in patients with T1D, and the results of these have varied. ${ }^{13} 1416-1830$ In Denmark, a case-control study of 124655 participants who had experienced fracture and 373962 controls showed that a prior episode of hypoglycemia significantly increased the risk of hip fracture (HR $1.55)$, although this study did not distinguish the type of diabetes present in its analysis of the relationship with hypoglycemia. ${ }^{16}$ Most recently, a nested case-control study that used the Danish National Patient Registry showed that hypoglycemia significantly increased fracture risk (OR 1.58) in patients with T1D. ${ }^{17}$ However, no significant relationship was found between hypoglycemia and fracture risk in a prospective study of 121 patients with T1D that was conducted in Australia, ${ }^{18}$ the Health Improvement Network (THIN) database of 5368 people with $\mathrm{T} 1 \mathrm{D}^{14}$ or a recent cross-sectional study of 600 patients with T1D that was conducted in three institutions in Italy. ${ }^{13}$ The present study is one of the few studies that has shown a significant association between hypoglycemia and fracture risk in a relatively small sample. This may be because we were able to obtain detailed clinical information and carefully define severe hypoglycemia, having conducted the study in single clinic, and therefore we could assess the relationship more precisely than could the investigators in the previous multicenter studies.

\section{Severe hypoglycemia and fractures in T2DM}

With respect to T2D, a retrospective study of 361210 Medicare-covered patients with T2D aged $\geq 65$ years in the USA showed that hypoglycemia is independently associated with a higher risk of fall-related fracture (OR 


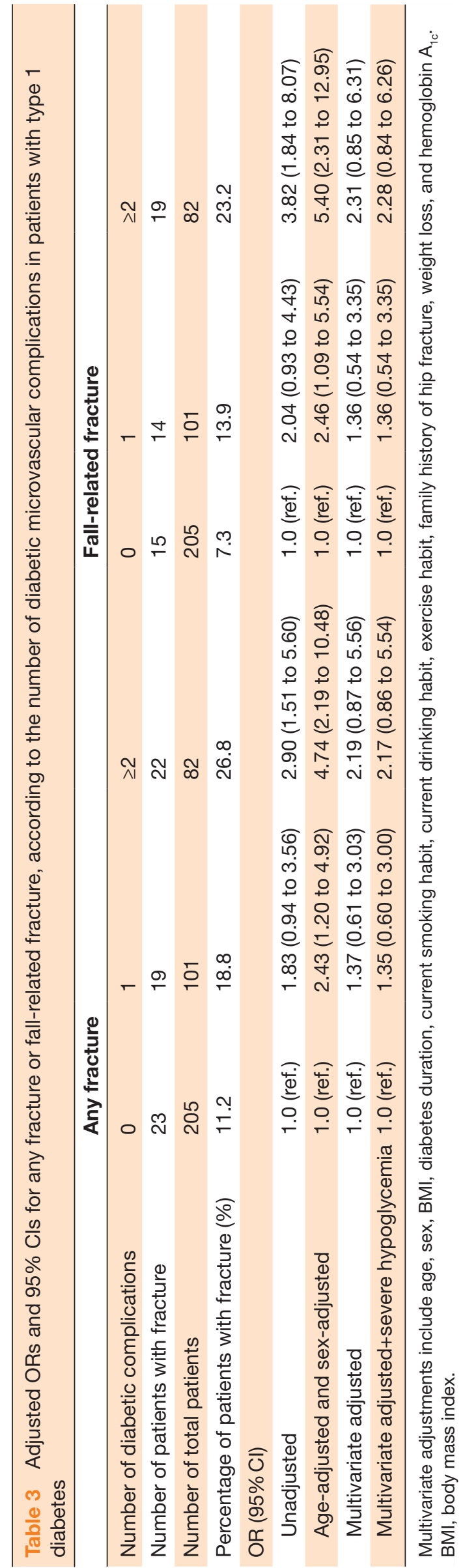

1.70). ${ }^{32}$ Another retrospective study that used the Taiwan National Health Insurance Research Database demonstrated that a history of severe hypoglycemia significantly increased the risk of hip fracture (HR 1.71) ${ }^{33}$ Furthermore, a study of 41163 patients with T2D showed that documented hypoglycemic events are a significant risk factor for fracture of any type (HR 1.20) and fragility fracture (HR 1.24). ${ }^{34}$ Most recently, multiple episodes of severe hypoglycemia have been reported to increase the risk of any type of fracture (HR 1.84) based on a study of 4706 Japanese patients with T2D. ${ }^{35}$ The magnitude of relative risk of fracture associated with hypoglycemia seems to be similar in T1D and T2D, based on previous studies, which suggests that the mechanisms of severe hypoglycemia-related fracture may be similar in patients with either of these types of diabetes.

\section{Mechanisms linking severe hypoglycemia and fractures}

The underlying mechanisms of severe hypoglycemiaassociated fracture risk are not fully understood. It has been shown that severe hypoglycemia increases the incidences of cognitive dysfunction, ${ }^{36}$ cardiovascular events, ${ }^{37}$ and falls,${ }^{38}$ which result in a higher incidence of fracture. ${ }^{39}$ However, only 4 of 92 fractures were assessed as being clearly hypoglycemia-related in the present study. Similarly, a cross-sectional study of 756 adults with T1D in the USA showed that only $3 \%$ of 322 patients that experienced non-osteoporotic fracture had symptoms of hypoglycemia at the time of fracture. These results might imply that few patients experienced falls or fractures due to ongoing severe hypoglycemia. ${ }^{30}$ Given that the present study was of relatively young patients who have lower risks of cognitive dysfunction or cardiovascular events, and few patients experienced fracture because of ongoing severe hypoglycemia, an indirect mechanism other than hypoglycemia-related falls is implicated. One of the potential links between hypoglycemia and higher risk of fracture might be hormones that are secreted in response to hypoglycemia, such as cortisol ${ }^{40}$ or epinephrine. ${ }^{41}$ Both of these hormones have been reported to induce bone fragility and increase fracture risk. ${ }^{42}$

The possible involvement of impaired awareness of hypoglycemia is another important consideration regarding the impact of hypoglycemia. Because impaired awareness of hypoglycemia is often overlooked, the number of episodes of hypoglycemia tends to be underestimated. ${ }^{43}$ It may at least explain the failure of some previous studies to show an association between hypoglycemia and fracture risk. Because severe hypoglycemia is strongly associated with impaired awareness of hypoglycemia, ${ }^{44}$ we were able to assess the relationship between hypoglycemia and fracture risk more accurately in the present study than in these previous studies. Indeed, $40 \%$ of the participants with a history of any type of fracture after the diagnosis of T1D reported that they had experienced impaired awareness of hypoglycemia (data not shown). 
Table 4 Adjusted ORs and 95\% Cls of risk factors for any fracture and fall-related fracture in patients with type 1 diabetes

\begin{tabular}{|c|c|c|}
\hline \multirow[b]{2}{*}{ Variables } & \multicolumn{2}{|c|}{ Multivariate-adjusted OR $(95 \% \mathrm{Cl})$} \\
\hline & Any fracture & Fall-related fracture \\
\hline Current smoking habit (yes vs no) & 2.88 (1.28 to 6.42$)$ & 2.21 (0.86 to 5.41$)$ \\
\hline Family history of hip fracture (yes vs no) & $2.50(0.51$ to 9.74$)$ & $4.81(0.94$ to 19.72$)$ \\
\hline Duration of diabetes (per 10 years increase) & $2.42(1.75$ to 3.43$)$ & $2.63(1.85$ to 3.85$)$ \\
\hline Severe hypoglycemia (yes vs no) & $2.10(1.11$ to 4.06$)$ & $1.93(0.94$ to 4.04$)$ \\
\hline Sex (male vs female) & 1.51 (0.78 to 2.92$)$ & $0.98(0.46$ to 2.07$)$ \\
\hline BMI (per 1 kg/m² increase) & $1.04(0.95$ to 1.14$)$ & 0.98 (0.88 to 1.08$)$ \\
\hline Exercise habit (yes vs no) & $1.03(0.56$ to 1.94$)$ & 0.85 (0.43 to 1.72$)$ \\
\hline Body weight change (per 10\% decrease) & $0.88(0.66$ to 1.11$)$ & $0.76(0.50$ to 1.02$)$ \\
\hline $\mathrm{HbA}_{1 \mathrm{c}}$ (per $1 \%$ increase) & 0.85 (0.59 to 1.20$)$ & 1.08 (0.71 to 1.62$)$ \\
\hline Age (per 10 years increase) & $0.79(0.62$ to 1.01$)$ & $0.80(0.60$ to 1.05$)$ \\
\hline Current alcohol habit (yes vs no) & $0.78(0.32$ to 1.81$)$ & 1.64 (0.64 to 4.01$)$ \\
\hline
\end{tabular}

Multivariate adjustments include all variables above.

$\mathrm{BMI}$, body mass index; $\mathrm{HbA}_{1 \mathrm{c}}$, hemoglobin $\mathrm{A}_{1 \mathrm{c}}$.

\section{Diabetic complications and fractures}

In the present study, more patients experienced any fracture or fall-related fracture if they had longer duration of diabetes, which is consistent with the results of previous studies of patients with T1D. ${ }^{30}$ A long duration of diabetes results in longer exposure to hyperglycemia, which causes bone fragility, ${ }^{14}$ and increases the prevalence of diabetic microvascular and macrovascular complications. Neuropathy has been reported to be associated with fracture risk ${ }^{45}$ via a higher risk of falls. ${ }^{46}$ Diabetic retinopathy has also been shown to be associated with higher fracture risk, ${ }^{13}$ possibly because of an increase in visual impairment-related fall risk. ${ }^{47}$ Finally, DKD has also been reported to be associated with higher fracture risk, ${ }^{12}$ possibly because of renal osteodystrophy. ${ }^{48}$ In the present study, neuropathy, diabetic retinopathy, and DKD were significantly associated with higher risks of fracture in the age-and sex-adjusted model, although the significance disappeared after multivariate adjustment, including for diabetes duration or glycemic control (online supplemental tables 2-4). Further studies are needed to elucidate whether each diabetic complication is an independent risk factor for fracture, or not, because previous studies have not always adjusted for the duration of diabetes or hypoglycemia, which are strongly correlated with the incidence of diabetic complications. Because of the recent advances in the therapy for diabetes and its complications, fewer patients now develop diabetic complications. Therefore, large-scale studies are needed to investigate the influence of specific factors on the incidence of diabetic complications.

\section{Strength and limitation}

The main strength of the present study was that we conducted the entire study in a single center in Asia, where the incidence of T1D is lower than in Western countries, which enabled us to obtain detailed information, including regarding severe hypoglycemia, diabetic complications, and the sites of fractures using the same criteria. However, this study had several limitations. First, fractures were assessed using a self-reported questionnaire. When the accuracy of the self-administered questionnaire was evaluated in 57 of the 64 patients who responded that they experienced fracture through re-interview by a trained practitioner, all of the patients provided details about the situation connected with the fracture. Second, the history of severe hypoglycemia was also assessed using a self-reported questionnaire. Nevertheless, assuming that some of the participants would have forgotten their history of severe hypoglycemia, the likely impact of this misclassification would be to attenuate any association with fracture risk. Third, we did not assess bone mineral density (BMD). However, BMD has been reported to underestimate fracture risk in patients with diabetes, and especially T1D. ${ }^{49}$ Thus, predictors for fracture other than BMD are needed to help prevent bone fracture in these patients. Fourth, the present study was of a smaller sample of patients with T1D to those of previous studies, ${ }^{13} 30$ which led to few fragility fractures, such as those of the femur/hip or spine, being recorded. However, it is difficult to recruit large numbers of older patients with T1D using clinic-based or hospital-based registries. Furthermore, a meta-analysis of observational studies has shown that a history of any type of fracture almost doubles the future risk of hip or osteoporotic fractures ${ }^{50}$ which means that investigation of the risk factors for any type of fracture may predominantly elucidate the risks for fragility fractures. Fifth, there were few patients with severe kidney disease in the present study, meaning that we could not fully investigate the influence of DKD on fracture risk. Sixth, because all the participants in the study were Japanese and because the study was conducted at a single center, it is unclear whether 
the conclusions can be generalized to other populations. Finally, we cannot draw conclusions regarding cause-andeffect relationships because of the cross-sectional design of the study.

\section{CONCLUSIONS}

In conclusion, the present study has demonstrated that severe hypoglycemia is significantly associated with a higher risk of incident bone fracture in Japanese patients with T1D, which is independent of the duration of diabetes or the presence of diabetic complications. Thus, to reduce fracture risk in patients with T1D, careful treatment for the prevention of diabetic complications and of severe hypoglycemia should be needed.

Acknowledgements The authors thank all medical and administrative staffs in Clinic Masae Minami. The authors also thank Drs Hiroaki Ooboshi, Masanori Tokumoto, Hiroki Fujii, Yoji Yoshikawa, Masatoshi Hara (Fukuoka Dental College), Udai Nakamura (Steel Memorial Yawata Hospital), Masahito Yoshinari (Kyushu Dental University), Ayaka Oshiro, Yutaro Oku, Taiki Higashi, Shunya Tanaka, and Tomohiro Nagasue (Kyushu University). Finally, the authors thank Edanz Group (https://en-author-services.edanz.com) for editing drafts of this manuscript.

Contributors YK, MM, and YM were responsible for the study concept and design. YK conducted the analysis and MM, YM, RY, TO, and TK interpreted the data and contributed to discussion. YK drafted the manuscript. All authors participated in critically revising the manuscript and approved the final version. YK and MM are the guarantors for this work and, as such, had full access to all the data in the study and take responsibility for the integrity of the data and the accuracy of the data analysis.

Funding This work was supported in part by The Japan Society for the Promotion of Science KAKENHI from the Ministry of Education, Culture, Sports, Science and Technology of Japan (grant numbers 20K19663 to YK), a Junior Scientist Development Grant supported by the Japan Diabetes Society (to YK), a Lilly Research Grant Program for Bone \& Mineral Research supported by the Japan Osteoporosis Foundation (to YK), and the Nakatomi Foundation (to YK).

Competing interests None declared.

Patient consent for publication Not required.

Ethics approval The Clinic Masae Minami Institutional Review Board approved the study (number MERC-19-001, dated October 1, 2019) and written informed consent was obtained from all the participants. The study was conducted according to the principles of the Declaration of Helsinki and its amendments.

Provenance and peer review Not commissioned; externally peer reviewed.

Data availability statement Data are available on reasonable request. The datasets generated during and/or analyzed during the current study are available from the corresponding author on reasonable request.

Supplemental material This content has been supplied by the author(s). It has not been vetted by BMJ Publishing Group Limited (BMJ) and may not have been peer-reviewed. Any opinions or recommendations discussed are solely those of the author(s) and are not endorsed by BMJ. BMJ disclaims all liability and responsibility arising from any reliance placed on the content. Where the content includes any translated material, BMJ does not warrant the accuracy and reliability of the translations (including but not limited to local regulations, clinical guidelines, terminology, drug names and drug dosages), and is not responsible for any error and/or omissions arising from translation and adaptation or otherwise.

Open access This is an open access article distributed in accordance with the Creative Commons Attribution Non Commercial (CC BY-NC 4.0) license, which permits others to distribute, remix, adapt, build upon this work non-commercially, and license their derivative works on different terms, provided the original work is properly cited, appropriate credit is given, any changes made indicated, and the use is non-commercial. See: http://creativecommons.org/licenses/by-nc/4.0/.
REFERENCES

1 Guariguata L, Whiting DR, Hambleton I, et al. Global estimates of diabetes prevalence for 2013 and projections for 2035. Diabetes Res Clin Pract 2014;103:137-49.

2 Patterson CC, Harjutsalo V, Rosenbauer J, et al. Trends and cyclical variation in the incidence of childhood type 1 diabetes in 26 European centres in the 25 year period 1989-2013: a multicentre prospective registration study. Diabetologia 2019;62:408-17.

3 Gregg EW, Li Y, Wang J, et al. Changes in diabetes-related complications in the United States, 1990-2010. N Engl J Med 2014;370:1514-23.

4 Livingstone SJ, Levin D, Looker HC, et al. Estimated life expectancy in a Scottish cohort with type 1 diabetes, 2008-2010. JAMA 2015;313:37-44.

5 Kirkman MS, Briscoe VJ, Clark N, et al. Diabetes in older adults. Diabetes Care 2012;35:2650-64.

6 Starup-Linde J, Hygum K, Harsløf T, et al. Type 1 diabetes and bone fragility: links and risks. Diabetes Metab Syndr Obes 2019;12:2539-47.

7 Janghorbani M, Van Dam RM, Willett WC, et al. Systematic review of type 1 and type 2 diabetes mellitus and risk of fracture. Am J Epidemiol 2007;166:495-505.

8 Wang $\mathrm{H}, \mathrm{Ba} \mathrm{Y}$, Xing Q, et al. Diabetes mellitus and the risk of fractures at specific sites: a meta-analysis. BMJ Open 2019:9:e024067.

9 Center JR, Nguyen TV, Schneider D, et al. Mortality after all major types of osteoporotic fracture in men and women: an observational study. Lancet 1999;353:878-82.

10 Johnell O, Kanis JA, Odén A, et al. Mortality after osteoporotic fractures. Osteoporos Int 2004:15:38-42.

11 Hough FS, Pierroz DD, Cooper C, et al. Mechanisms in endocrinology: mechanisms and evaluation of bone fragility in type 1 diabetes mellitus. Eur J Endocrinol 2016;174:R127-38.

12 Vestergaard P, Rejnmark L, Mosekilde L. Diabetes and its complications and their relationship with risk of fractures in type 1 and 2 diabetes. Calcif Tissue Int 2009;84:45-55.

13 Leanza G, Maddaloni E, Pitocco D, et al. Risk factors for fragility fractures in type 1 diabetes. Bone 2019;125:194-9.

14 Thayakaran R, Perrins M, Gokhale KM, et al. Impact of glycaemic control on fracture risk in 5368 people with newly diagnosed type 1 diabetes: a time-dependent analysis. Diabet Med 2019;36:1013-9.

15 Vavanikunnel J, Charlier S, Becker C, et al. Association between glycemic control and risk of fracture in diabetic patients: a nested case-control study. J Clin Endocrinol Metab 2019;104:1645-54.

16 Vestergaard P, Rejnmark L, Mosekilde L. Relative fracture risk in patients with diabetes mellitus, and the impact of insulin and oral antidiabetic medication on relative fracture risk. Diabetologia 2005:48:1292-9.

17 Jensen MH, Vestergaard P. Hypoglycaemia and type 1 diabetes are associated with an increased risk of fractures. Osteoporos Int 2019;30:1663-70.

18 Hamilton EJ, Davis WA, Bruce DG, et al. Risk and associates of incident hip fracture in type 1 diabetes: the Fremantle diabetes study. Diabetes Res Clin Pract 2017;134:153-60.

19 DIAMOND Project Group. Incidence and trends of childhood type 1 diabetes worldwide 1990-1999. Diabet Med 2006;23:857-66.

20 Weng J, Zhou Z, Guo L, et al. Incidence of type 1 diabetes in China, 2010-13: population based study. BMJ 2018;360:j5295.

21 Worldometers. Available: https://www.worldometers.info/worldpopulation/asia-population/ [Accessed 05 Mar 2020].

22 Kuzuya T, Nakagawa S, Satoh J, et al. Report of the Committee on the classification and diagnostic criteria of diabetes mellitus. Diabetes Res Clin Pract 2002;55:65-85.

23 American Diabetes Association. 2. Classification and Diagnosis of Diabetes: Standards of Medical Care in Diabetes-2020. Diabetes Care 2020:43:S14-31.

24 Komorita Y, Iwase M, Fujii H, et al. Impact of body weight loss from maximum weight on fragility bone fractures in Japanese patients with type 2 diabetes: the Fukuoka diabetes registry. Diabetes Care 2018;41:1061-7.

25 Workgroup on Hypoglycemia, American Diabetes Association. Defining and reporting hypoglycemia in diabetes: a report from the American diabetes association Workgroup on hypoglycemia. Diabetes Care 2005;28:1245-9.

26 Seino Y, Nanjo K, Tajima N, et al. Report of the Committee on the classification and diagnostic criteria of diabetes mellitus. Diabetol Int 2010;1:2-20.

27 Matsuo S, Imai E, Horio M, et al. Revised equations for estimated GFR from serum creatinine in Japan. Am J Kidney Dis 2009;53:982-92. 
28 Yasuda H, Sanada M, Kitada K, et al. Rationale and usefulness of newly devised abbreviated diagnostic criteria and staging for diabetic polyneuropathy. Diabetes Res Clin Pract 2007;77 Suppl 1:S178-83.

29 American Diabetes Association. 11. Microvascular Complications and Foot Care: Standards of Medical Care in Diabetes-2021. Diabetes Care 2021;44:S151-67.

30 Dhaliwal R, Foster NC, Boyle C, et al. Determinants of fracture in adults with type 1 diabetes in the USA: results from the T1D exchange clinic registry. J Diabetes Complications 2018;32:1006-11.

31 Fink HA, Milavetz DL, Palermo L, et al. What proportion of incident radiographic vertebral deformities is clinically diagnosed and vice versa? J Bone Miner Res 2005;20:1216-22.

32 Johnston SS, Conner C, Aagren M, et al. Association between hypoglycaemic events and fall-related fractures in Medicarecovered patients with type 2 diabetes. Diabetes Obes Metab 2012;14:634-43.

33 Hung YC, Lin CC, Chen HJ, et al. Severe hypoglycemia and hip fracture in patients with type 2 diabetes: a nationwide populationbased cohort study. Osteoporos Int 2017;28:2053-60.

34 Ntouva A, Toulis KA, Keerthy D. Hypoglycaemia is associated with increased risk of fractures in patients with type 2 diabetes mellitus: a cohort study. Eur J Endocrinol 2018.

35 Komorita Y, Iwase M, Fujii H, et al. Both hypo- and hyperglycaemia are associated with increased fracture risk in Japanese people with type 2 diabetes: the Fukuoka Diabetes Registry. Diabet Med 2020;37:838-47.

36 Whitmer RA, Karter AJ, Yaffe K, et al. Hypoglycemic episodes and risk of dementia in older patients with type 2 diabetes mellitus. JAMA 2009;301:1565-72.

37 Zoungas S, Patel A, Chalmers J, et al. Severe hypoglycemia and risks of vascular events and death. N Engl J Med 2010;363:1410-8.

38 Shah VN, Wu M, Foster N, et al. Severe hypoglycemia is associated with high risk for falls in adults with type 1 diabetes. Arch Osteoporos 2018;13:66.
39 Scandol JP, Toson B, Close JCT. Fall-Related hip fracture hospitalisations and the prevalence of dementia within older people in New South Wales, Australia: an analysis of linked data. Injury 2013;44:776-83.

40 Dotson S, Freeman R, Failing HJ, et al. Hypoglycemia increases serum interleukin-6 levels in healthy men and women. Diabetes Care 2008;31:1222-3.

41 Tesfaye N, Seaquist ER. Neuroendocrine responses to hypoglycemia. Ann N Y Acad Sci 2010;1212:12-28.

42 Hardy R, Cooper MS. Adrenal gland and bone. Arch Biochem Biophys 2010;503:137-45.

43 Geddes J, Wright RJ, Zammitt NN, et al. An evaluation of methods of assessing impaired awareness of hypoglycemia in type 1 diabetes. Diabetes Care 2007;30:1868-70.

44 Flatt AJS, Little SA, Speight J, et al. Predictors of recurrent severe hypoglycemia in adults with type 1 diabetes and impaired awareness of hypoglycemia during the HypoCOMPaSS study. Diabetes Care 2020;43:44-52.

45 Rix M, Andreassen H, Eskildsen P. Impact of peripheral neuropathy on bone density in patients with type 1 diabetes. Diabetes Care 1999;22:827-31.

46 Brown SJ, Handsaker JC, Bowling FL, et al. Diabetic peripheral neuropathy compromises balance during daily activities. Diabetes Care 2015;38:1116-22.

47 Gupta P, Aravindhan A, Gand ATL, et al. Association between the severity of diabetic retinopathy and falls in an Asian population with diabetes: the Singapore epidemiology of eye diseases study. JAMA Ophthalmol 2017;135:1410-6.

48 Aleksova J, $\mathrm{Ng} \mathrm{KW}$, Jung C, et al. Bone health in chronic kidney disease-mineral and bone disorder: a clinical case seminar and update. Intern Med J 2018;48:1435-46.

49 Vestergaard P. Discrepancies in bone mineral density and fracture risk in patients with type 1 and type 2 diabetes--a meta-analysis. Osteoporos Int 2007;18:427-44.

50 Kanis JA, Johnell O, De Laet C, et al. A meta-analysis of previous fracture and subsequent fracture risk. Bone 2004;35:375-82. 